\title{
Patient-reported outcomes in a large North American cohort living with chronic hepatitis $B$ virus: a cross-sectional analysis
}

\author{
Donna M. Evon ${ }^{1}$ (D) | Hsing-Hua S. Lin ${ }^{2}$ | Mandana Khalili ${ }^{3}$ (D) | Robert J. Fontana \\ Colina Yim $^{5}$ | Abdus S. Wahed ${ }^{2} \mid$ Michael W. Fried $^{1} \mid$ Jay H. Hoofnagle ${ }^{6}$ for the \\ Hepatitis B Research Network (HBRN)*
}

${ }^{1}$ Department of Medicine, University of North Carolina, Chapel Hill, NC, USA

${ }^{2}$ Departments of Epidemiology and Biostatistics, University of Pittsburgh, Pittsburgh, PA, USA

${ }^{3}$ Department of Medicine, University of California, San Francisco, CA, USA

${ }^{4}$ Department of Internal Medicine, University of Michigan, Ann Arbor, MI, USA

${ }^{5}$ Toronto Centre for Liver Disease, University of Toronto, Toronto, Canada

${ }^{6}$ Liver Disease Research Branch, NIDDK, $\mathrm{NIH}$, Bethesda, MD, USA

\section{Correspondence}

Donna M. Evon, Department of Medicine, University of North Carolina, Chapel Hill NC, USA.

Email: donna_evon@med.unc.edu

Funding information

The HBRN was funded as a Cooperative Agreement between the National Institute of Diabetes and Digestive and Kidney Diseases (NIDDK) and the following investigators: Lewis R. Roberts, MB, ChB, PhD (U01-DK082843), Anna Suk-Fong Lok, MD (U01-DK082863), Steven H. Belle, PhD, MScHyg (U01-DK082864), Kyong-Mi Chang, MD (U01-DK082866), Michael W. Fried, MD (U01-DK082867), Adrian M. Di Bisceglie, MD (U01-DK082871), William M. Lee, MD (U01-DK082872), Harry L. A. Janssen, MD, PhD (U01-DK082874), Daryl T-Y Lau, MD, MPH (U01-DK082919), Richard K. Sterling, MD, MSc (U01-DK082923), Steven-Huy B. Han, MD (U01-DK082927), Robert C. Carithers, MD (U01-DK082943), Mandana Khalili, MD (U01-DK082944), an interagency agreement with NIDDK: Lilia M. GanovaRaeva, PhD (A-DK-3002-001) and support from the intramural program, NIDDK, NIH: Marc G. Ghany, MD. Additional funding to

\section{Summary}

Background: Patient-reported outcomes (PROs) such as health-related quality of life (HRQoL) and symptoms associated with chronic hepatitis B viral (HBV) infection have not been well-described in North American cohorts.

Aims: To evaluate several PROs and associations with HBV disease activity markers. Methods: Cross-sectional analysis including 876 adults who completed PRO measures during the Hepatitis B Research Network Adult Cohort Study. Participants on HBV treatment were excluded. Outcomes included: HRQoL using the SF-36 mental component summary and physical component summary scores; symptom burden using a 10-item Total Symptom Checklist and fatigue using an instrument from the PatientReported Outcomes Measurement Information System ${ }^{\circledR}$. Covariates included laboratory markers of disease severity, virological status, comorbidities and medications.

Results: Median age was 42 (range: 19-79), 51\% were female, 73\% Asian, 19\% HBeAg $(+), 2 \%$ had AST-platelet ratio index (APRI) $\geq 1.5$ and $74 \%$ without comorbidities. Mean mental component summary $\mathrm{T}$-score $=52$, physical component summary $\mathrm{T}$-score $=54$ and PROMIS Fatigue T-score $=47$. On a scale from 0 (none) to 40 (extreme), the mean Symptom Checklist score $=3$ and $25 \%$ reported no symptoms. The most frequent symptoms were fatigue (60\%), irritability (32\%) and itching (32\%). Most symptoms were 'a little bit' bothersome. In multivariable regressions, APRI $\geq 1.50$ and more comorbidities were associated with worse patient-reported outcomes; virological markers were not. Adding the Total Symptom Checklist score to original regression models increased explanation of variation in the mental component summary score from $4 \%$ to $44 \%$ and the Physical Component Summary Score from $17 \%$ to $34 \%$.

Conclusions: Untreated North American HBV patients with mild liver disease report favourable health-related quality of life and minimal symptoms. HBV does not impact health-related quality of life unless advanced liver disease or comorbidities are present. High symptom burden explains substantial variation in health-related quality of life. (CT.gov identifier: NCT01263587). 
support this study was provided to KyongMi Chang, MD, the Immunology Center, (NIH/NIDDK Center of Molecular Studies in Digestive and Liver Diseases P30DK50306, NIH Public Health Service Research Grant M01-RR00040), Richard K. Sterling, MD, MSc (UL1TR000058, NCATS (National Center for Advancing Translational Sciences, NIH), Norah A. Terrault, MD, MPH (CTSA Grant Number UL1TR000004), Michael W. Fried, MD (CTSA Grant Number UL1TR001111) and Anna Suk-Fong Lok (CTSA Grant Number UL1RR024986, U54TR001959.) Additional support was provided by Gilead Sciences, Inc. and Roche Molecular Systems via a CRADA through the NIDDK.

\section{1 | INTRODUCTION}

Chronic hepatitis B viral (HBV) infection affects over one million people living in the United States and Canada and is estimated to lead to advanced cirrhosis or liver cancer in $10 \%$ of infected individuals. ${ }^{1}$ Health-related quality of life (HRQoL) refers to patients' perceptions of their overall health status, including their physical, mental, psychological and social functioning and ability to engage in life activities and responsibilities. Several studies have demonstrated that HRQoL may be compromised in patients chronically infected with HBV compared to population controls. ${ }^{2}$ Decrements in $\mathrm{HRQ}$ oL are often associated with liver disease severity, such that patients with compensated or decompensated cirrhosis or hepatocellular carcinoma tend to have worse HRQoL. ${ }^{2-6}$ However, it is less clear whether patients with chronic HBV infection without cirrhosis or cancer have worse HRQoL compared to the general population. ${ }^{2,5,7-11}$ Studies have also inadequately accounted for different phenotypes of chronic hepatitis $B$, such as subgroups who are immune tolerant, have active disease or are inactive HBsAg carriers. ${ }^{12}$ The majority of studies of HRQoL in patients with chronic HBV infection have been conducted in Asia, the Middle East or Europe, where sociocultural differences likely impact study findings, making generalisability to North American cohorts challenging. Previous studies have also been limited by small sample sizes, inclusion of a minimal number of clinical or disease factors, and diverse patient populations that included both those with chronic hepatitis $B$ and those with other types of liver diseases.

We hypothesised that investigating other salient patient-reported outcomes may enhance our understanding of the physical and emotional sequelae of living with chronic hepatitis B including symptoms such as fatigue, depression and pruritus. Fatigue has been investigated in several studies, but is generally nonspecific and is affected by other, unrelated physical, mental and emotional factors. $^{3,13,14}$ The paucity of data related to symptoms may be in part due to lack of instruments to adequately measure symptom severity in patients with chronic HBV infection. ${ }^{3}$ Measures of symptom presence and severity may be potentially more sensitive to variations in
HBV disease markers or activity, compared to a broad nonspecific measure of HRQoL.

The Hepatitis B Research Network provides a unique opportunity to evaluate symptom burden and HRQoL in a large multi-ethnic North American population. ${ }^{15}$ Our aims were to fully characterise symptoms and HRQoL in a cohort of adults with chronic HBV infection and to evaluate the association between the patient-reported outcomes and liver disease severity and clinical markers. We also examined the relationship among three patient-reported outcome instruments: the Patient-Reported Outcomes Measurement Information System (PROMIS ${ }^{\circledR}$ ) Fatigue 7a-item instrument, a multi-symptom checklist and the SF-36v2 ${ }^{\mathrm{TM}}$ Health Survey.

\section{2 | MATERIALS AND METHODS}

\section{1 | Study design}

The current analysis is a cross-sectional study of patients diagnosed with chronic HBV infection and enrolled in the Hepatitis B Research Network Adult Cohort study. The Hepatitis B Research Network is a consortium of investigators from 21 geographically diverse adult clinical centres across the United States and Canada funded by a cooperative agreement between the principal investigators and the National Institute of Diabetes and Digestive and Kidney Diseases of the National Institutes of Health. All protocols were approved by the Steering Committee and the Institutional Review Boards or Research Ethics Boards of the participating sites. All participants provided written informed consent. The Hepatitis B Research Network Adult Cohort Study is registered on ClinicalTrials.gov (NCT01263587).

\section{2 | Participants}

The Hepatitis B Research Network Adult Cohort Study enrolled 2,032 adults with chronic hepatitis B not on any HBV therapy, from 


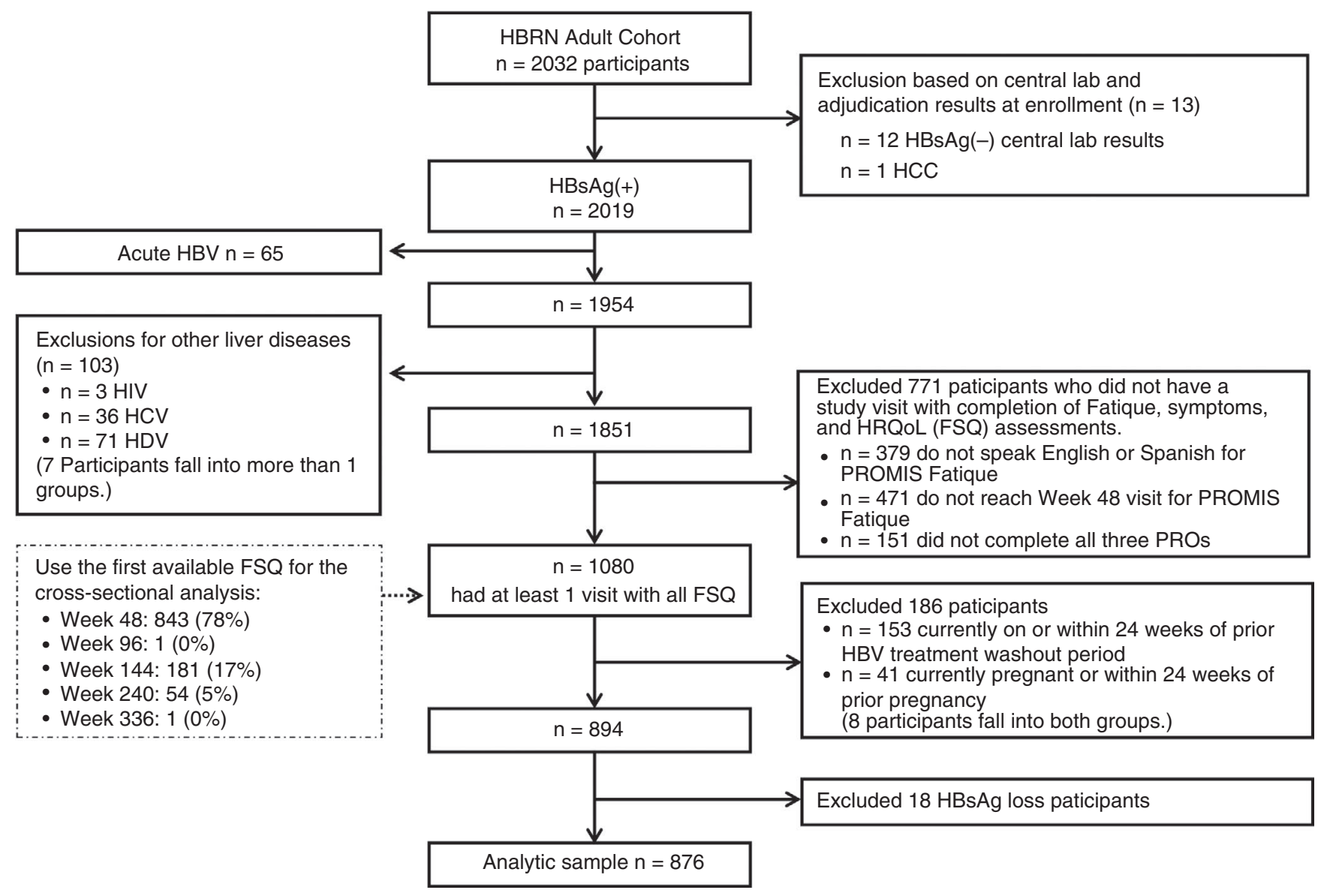

FIGURE 1 Study flow chart

2011 to 2018. After excluding 12 participants who tested $\mathrm{HBsAg}$ negative at the central laboratory and one participant adjudicated for hepatocellular carcinoma at enrollment, there were 2,019 HBsAg positive eligible participants (Figure 1). In addition, participants were excluded from this analysis if they had acute HBV infection, coinfection with human immunodeficiency virus, hepatitis $C$ virus or hepatitis D virus, were pregnant or were on HBV therapy within the 6 months before completing the patient-reported outcome instruments, or were unable to speak or read English or Spanish. The final analytic sample included 876 study participants age 18 or older who completed all three patient-reported outcome instruments (available in English or Spanish) at the same research visit and who were not receiving HBV therapy for the prior 6 months. Each participant's first assessment of all three patient-reported outcome instruments was used in this analysis.

\section{3 | Patient-reported outcome measures}

\subsection{1 | Symptom checklist (SCL)}

The SCL is a 10 -item instrument initially developed by the National Institute of Diabetes and Digestive and Kidney Diseases to evaluate the symptoms of chronic hepatitis C. Participants were asked,
'During the last month, how much have you been bothered by the following symptom: fatigue, itching, pain over liver, irritability, depression/sadness, nausea, poor appetite, weight loss, dark urine, jaundice'. For each symptom, the participant marked a box to indicate how bothered they were by the symptom in the last month: None at all (0), A little bit (1), Moderately (2), Quite a bit (3) or Extremely (4). If they did not have the symptom, they marked 'None at all'. A total SCL score (T-SCL) was then created by summing the 10 symptom scores, with a possible range from 0 to 40 , with higher scores indicating worse symptoms. T-SCL was used as a measure of total symptom burden.

\subsection{2 | PROMIS ${ }^{\circledR}$ fatigue 7a}

The PROMIS Fatigue 7a-item instrument consists of seven questions that assessed the experience of fatigue and interference of fatigue on daily activities over the past 7 days. ${ }^{16,17}$ Participants respond on a 5-point ordinal scale (Never, Rarely, Sometimes, Often and Always) to the following questions: 'In the past 7 days, how often did you feel tired? How often did you experience extreme exhaustion? How often did you run out of energy? How often did your fatigue limit you at work (include work at home)? How often were you too tired to think clearly? How often were to too tired to take a bath or shower? How often did you have 
enough energy to exercise strenuously'. The total raw score is translated into a standardised T-score, with a mean of 50 and a standard deviation of 10 . Higher scores indicate worse fatigue.

\subsection{3 $\mid$ SF-36v2 ${ }^{\text {TM }}$ health survey}

The SF-36 is a widely used, generic measure o HRQOL. ${ }^{18,19}$ The SF-36 consists of items that load on eight subscales, which then can be combined into two summary scores, a physical component summary (PCS) and a mental component summary (MCS). The PCS and MCS are converted to T-scores based on population-based norms with each scale having a mean score of 50 and standard deviation of 10 . Higher scores indicate better physical and mental health.

\section{4 | Sociodemographic covariates}

The following variables collected at the baseline visit into the cohort study were used in these analyses: age, sex, race, place of birth and education.

\subsection{Clinical and virological covariates}

The following clinical and virological data collected at the same visit as the patient-reported outcomes instruments were used to evaluate factors associated with the patient-reported outcomes: serum alanine aminotransferase (ALT), aspartate aminotransferase (AST), AST-platelet ratio index (APRI), albumin, cirrhosis status, HBV phenotypes, HBV DNA $\left(\log _{10} \mathrm{IU} / \mathrm{mL}\right), \mathrm{HBeAg}$ (positive, negative), quantitative $\mathrm{HBsAg}$ (qHBsAg $\log _{10} \mathrm{IU} / \mathrm{mL}$ ), number of medical comorbidities $(0,1,2+)$ and number of medications $(0,1,2+)$. The upper limit of normal for ALT was $30 \mathrm{U} / \mathrm{L}$ for males and $20 \mathrm{U} / \mathrm{L}$ for females. The HBV phenotypes were defined based on $\mathrm{HBeAg}$ status and HBV DNA and ALT levels as previously defined by the Hepatitis B Research Network. ${ }^{12}$ The definitions used were as follows: Inactive carriers had HBsAg without $\mathrm{HBeAg}$, normal ALT levels ( $\leq 1$ upper limit of normal) and no or low HBV DNA levels ( $\leq 10,000 \mathrm{IU} / \mathrm{mL})$. Immune-active chronic hepatitis $B$ was defined by the presence of $\mathrm{HBsAg}$ and elevated levels of ALT (>1 upper limit of normal) and HBV DNA $\geq 100,000 \mathrm{IU} / \mathrm{mL}$ in those with $\mathrm{HBeAg}$ and $>10,000 \mathrm{IU} / \mathrm{mL}$ in those without $\mathrm{HBeAg}$. Immune-tolerant chronic hepatitis $\mathrm{B}$ was defined by the presence of $\mathrm{HBeAg}$ and HBV DNA $\geq 100,000 \mathrm{IU} / \mathrm{mL}$ in serum, with normal ALT levels ( $\leq 1$ upper limit of normal). Patients not fitting into any of these three patterns were classified as having an 'Indeterminate' HBV phenotype.

\subsection{Statistical analyses}

Categorical data were summarised with frequencies and percentages and continuous data were summarised as median (25th and 75th percentiles). To evaluate the relationships among four patientreported outcome scores (T-SCL, PROMIS Fatigue, SF-36 MCS, SF-36 PCS), each total score was analysed as a continuous measure. Ordinal variables for each of the $10 \mathrm{SCL}$ symptoms on a 5-point ordinal scale were also created. Distribution of each outcome score was summarised using histograms. We computed Spearman correlation coefficients between the four outcome scores and between each outcome measure and continuous covariates. Unadjusted and adjusted associations between the outcome scores and demographics, HBV disease markers or clinical features were investigated using multiple linear regression model for Fatigue, MCS and PCS; negative binomial regression was used for T-SCL outcome due to its right-skewed nature. We first accounted for sociodemographics (sex, age, race, education) in the multivariable model and used stepwise regression models for variable selection based on Schwarz Bayesian Information Criterion. The criterion for both entry and removal from the models was with $P<.05$. $P$ values were adjusted for multiple comparisons across four outcome measures in multivariable models using the Bonferroni-Holm method. Exploratory regression models were conducted to determine if symptoms (total and individual) were independent predictors of MCS and PCS and could predict additional variation in MCS and PCS, after controlling for the covariates in the initial regression models. All analyses conducted in statistical software packages SAS (version 9.4; SAS Institute Inc, Cary, NC) and R (version 3.5.3; R Foundation for Statistical Computing, Vienna, Austria). All tests were two-sided and a $p$ value $<0.05$ was considered statistically significant.

\section{3 | RESULTS}

\section{1 | Study flowchart}

Of 2019 eligible HBsAg-positive adult participants enrolled in the Hepatitis B Research Network cohort study, 876 patients met inclusion criteria for the current analysis (Figure 1). Patients ( $n=168)$ were excluded who had acute HBV $(n=65)$ or co-infected with human immunodeficiency virus, hepatitis $C$ virus or hepatitis $D$ virus ( $n=103$ ); 771 participants were excluded who did not have complete outcome measure data at any time point, and 186 patients were excluded for being on HBV treatment or pregnant within 24 weeks prior to outcome assessments. The majority (78\%) of patients included in this analysis completed all PROs at week 48 of entry into the cohort study, followed by $17 \%$ at week 144 , and $5 \%$ at week 240 .

\section{2 | Participant characteristics}

The characteristics of the 876 participants are summarised in Table 1. The median age was 42 years. Men and women were equally represented. Patients were predominantly Asian (73\%) and 80\% were born outside of the United States and Canada. The majority (59\%) were 
TABLE 1 Demographic and clinical characteristics of participants

\begin{tabular}{|c|c|}
\hline Variables & All $(n=876)$ \\
\hline \multicolumn{2}{|l|}{ Age at Current Study Visit } \\
\hline Median(25th:75th) & $42.2(33.8: 52.0)$ \\
\hline Min:Max & 19.0:79.3 \\
\hline \multicolumn{2}{|l|}{ Sex } \\
\hline Male & 431 (49\%) \\
\hline Female & 445 (51\%) \\
\hline Race & $n=873$ \\
\hline White & $102(12 \%)$ \\
\hline Black & $102(12 \%)$ \\
\hline Asian & $638(73 \%)$ \\
\hline Other/Mixed & $31(4 \%)$ \\
\hline Education level & $n=870$ \\
\hline Below Bachelor & $357(41 \%)$ \\
\hline Bachelor or Higher & $513(59 \%)$ \\
\hline Place of birth & $n=874$ \\
\hline Outside of the US/Canada & 697 (80\%) \\
\hline In the US/Canada & $177(20 \%)$ \\
\hline Phenotype & $n=809$ \\
\hline Immune tolerant & $24(3 \%)$ \\
\hline $\mathrm{HBeAg}+\mathrm{CHB}$ & $118(15 \%)$ \\
\hline $\mathrm{HBeAg}-\mathrm{CHB}$ & $122(15 \%)$ \\
\hline Inactive carrier & $226(28 \%)$ \\
\hline Indeterminant & 319 (39\%) \\
\hline ALTxULN $($ male $=30$, female $=20 \mathrm{U} / \mathrm{L})$ & $n=844$ \\
\hline Median(25th:75th) & $1.2(0.9: 1.7)$ \\
\hline Min:Max & $0.3: 37.4$ \\
\hline ALTxULN & $\mathrm{n}=844$ \\
\hline$\leq 1$ ULN & 302 (36\%) \\
\hline$>1-3$ ULN & 480 (57\%) \\
\hline$>3-5$ ULN & $40(5 \%)$ \\
\hline$>5$ ULN & $22(3 \%)$ \\
\hline ASTxULN (laboratory-specific ULN) & $\mathrm{n}=842$ \\
\hline Median(25th:75th) & $0.7(0.5: 0.9)$ \\
\hline Min:Max & $0.3: 14.1$ \\
\hline ASTxULN & $\mathrm{n}=842$ \\
\hline Normal AST & 701 (83\%) \\
\hline Abnormal AST & 141 (17\%) \\
\hline $\mathrm{HBeAg}$ & $\mathrm{n}=835$ \\
\hline Negative & $675(81 \%)$ \\
\hline Positive & $160(19 \%)$ \\
\hline HBV DNA $\left(\log _{10} 1 \mathrm{U} / \mathrm{mL}\right)$ & $n=868$ \\
\hline Median(25th:75th) & $3.4(2.5: 5.0)$ \\
\hline Min:Max & <LLOD: 9.5 \\
\hline Quantifiable HBV DNA & $\mathrm{n}=868$ \\
\hline
\end{tabular}

(Continues)
TABLE 1 (Continued)

\begin{tabular}{|ll|}
\hline Variables & All ( $=876)$ \\
\hline$\geq 20 \mathrm{IU} / \mathrm{mL}$ & $802(92 \%)$ \\
\hline Quantitative HBsAg $\left(\log _{10} \mathrm{IU} / \mathrm{mL}\right)$ & $\mathrm{n}=823$ \\
\hline Median(25th:75th) & $3.4(2.6: 4.1)$ \\
\hline Min:Max & $<\mathrm{LLOD}: 5.5$ \\
\hline Albumin (g/dL) & $\mathrm{n}=823$ \\
\hline Median(25th:75th) & $4.3(4.1: 4.5)$ \\
\hline Min:Max & $3.1: 5.4$ \\
\hline APRI (AST-platelet ratio index) & $\mathrm{n}=714$ \\
\hline$\leq 0.50$ & $566(79 \%)$ \\
\hline$>0.50-1.50$ & $133(19 \%)$ \\
\hline$>1.50$ & $15(2 \%)$ \\
\hline Cirrhosis prior/at PRO visit & \\
\hline No & $863(99 \%)$ \\
\hline Yes & $13(1 \%)$ \\
\hline Number of medical comorbidities & \\
\hline 0 & $649(74 \%)$ \\
\hline 1 & $137(16 \%)$ \\
\hline $2+$ & $90(10 \%)$ \\
\hline Number of concomitant medications & $683(76 \%)$ \\
\hline 0 & $145(17 \%)$ \\
\hline 1 & $68 \%$ \\
\hline $2+$ & \\
\hline
\end{tabular}

Note: LLOD: Lower limit of detection (HBV DNA $=10 \mathrm{IU} / \mathrm{mL}=1 \log _{10}$ $\mathrm{IU} / \mathrm{mL}$, quantitative $\mathrm{HBsAg}=0.05 \mathrm{IU} / \mathrm{mL}=-1.3 \log _{10} \mathrm{IU} / \mathrm{mL}$ )

college educated or greater. For HBV phenotype, $28 \%$ were considered inactive carriers, $30 \%$ had active disease (15\% with $\mathrm{HBeAg}$ and $15 \%$ without $\mathrm{HBeAg}$ ), $3 \%$ were immune tolerant and $39 \%$ were designated as 'indeterminate'. ALT levels were raised (> 1 upper limit of normal) in $64 \%$ of participants but were largely in the range of one to three times the upper limit of normal. HBV DNA was quantifiable $(\geq 20 \mathrm{lU} / \mathrm{mL}$ ) in $92 \%$ of patients and the median HBV DNA level was 3.4 $\log _{10} \mathrm{IU} / \mathrm{mL}$ and majority (81\%) had HBeAg-negative status. Only $2 \%$ of participants had APRI scores $\geq 1.5$ suggestive of advanced fibrosis and less than $1 \%(n=13)$ had known cirrhosis. The majority of patients (74\%) had no medical comorbidities; only $10 \%$ had two or more comorbidities. The majority (76\%) were not taking concomitant medications.

\subsection{Distribution of study outcomes}

On a scale of 0 to 40, the mean T-SCL score was 3.1 (range: 0-26) (Figure 2). A significant floor effect was observed with $24.7 \%$ ( $n=216$ ) of patients reporting no symptoms of liver disease. Out of a possible range from 29.4 to 83.2 , the mean PROMIS Fatigue T-score was 46.8 (range: 29.4-72.9), which is lower (better) than the mean of the general population (defined as a T-score of 50). Out of a possible range from 0 to 100 , the MCS mean was 52.2 and the PCS mean was 
Total symptom score (T-SCL)
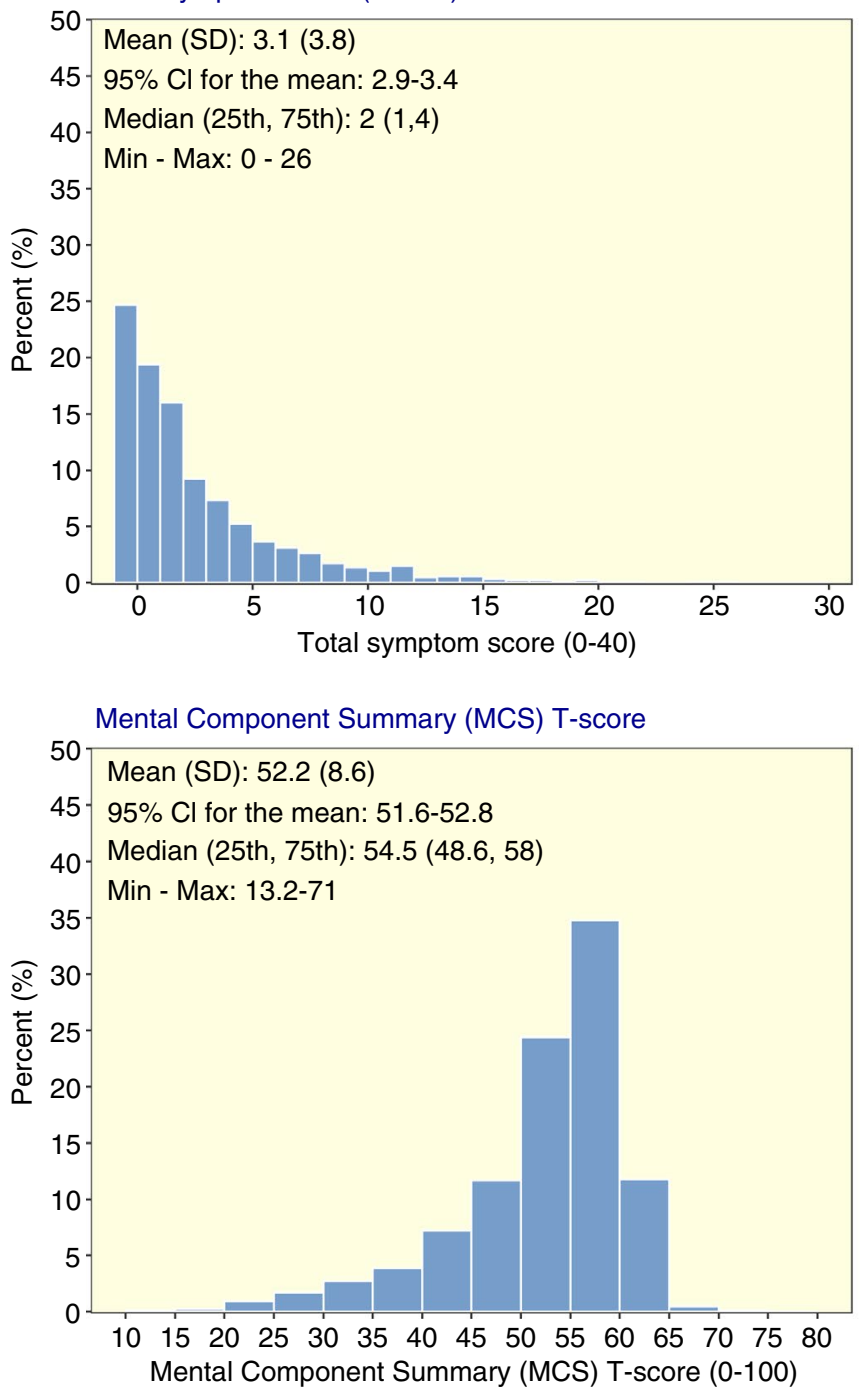

Fatigue T - score

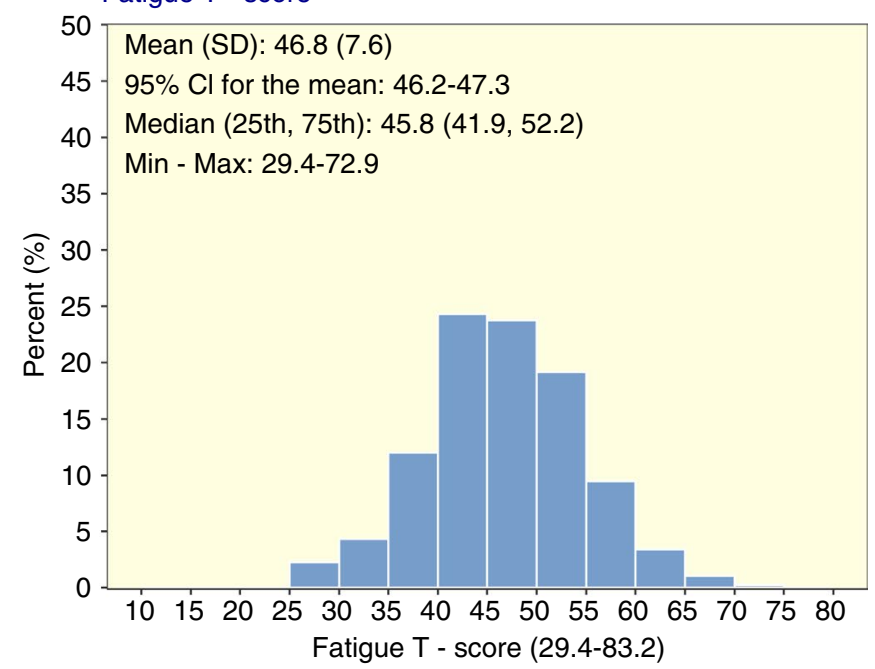

Physical Component Summary (PCS) T-score

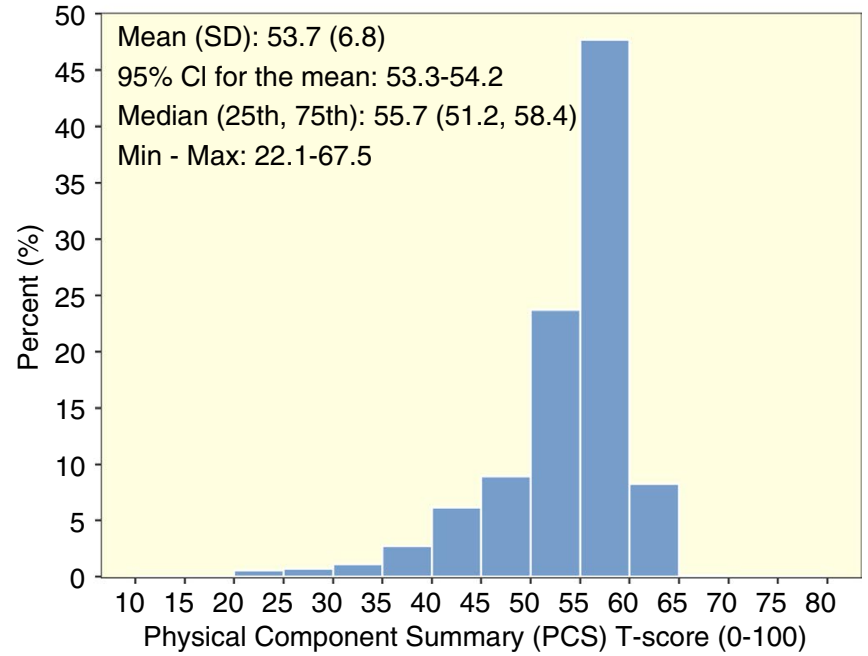

FIGURE 2 Distribution of each patient-reported outcome measure. Higher scores in the physical component summary score and mental component summary score indicate better physical and mental health. Higher scores in Fatigue indicate more fatigue. Higher Total Symptom Checklist scores indicate higher numbers of symptoms or more severe symptoms

53.7, which were both higher (better) than the general US population norms (T-score of 50).

Figure 3 displays the cumulative frequency for each of the 10 symptoms from the SCL instrument. Many patients indicated the absence of symptoms. Fatigue was the most frequent symptom with $60 \%$ of patients reporting fatigue that was 'a little bit' to 'extremely' bothersome, followed by irritability (32\%) and itching (32\%). Only $2 \%$ of patients reported jaundice. Most symptoms were rated only as 'A little bit' bothersome; rarely were symptoms rated 'Quite a bit' or 'Extremely' bothersome.

\section{4 | Associations among patient-reported outcome instruments}

The bivariate correlations among the MCS T-score, PCS T-score, PROMIS Fatigue T-score, T-SCL and the 10 symptom sub-scores generally ranged from $r=0.20$ to 0.50 (Figure S1). PCS was moderately correlated with PROMIS Fatigue $(r=0.40)$ and T-SCL $(r=0.40)$. MCS was strongly correlated with SCL-Depression/Sadness item ( $r=0.60)$, overall T-SCL score, SCL-Fatigue and PROMIS Fatigue (all $r$ 's $=0.50$ ). PROMIS Fatigue was moderately correlated with SCL-Depression/ Sadness, SCL-Irritability and SCL-Fatigue ( $r$ 's $=0.40-0.50$ ) in addition to the MCS and PCS. Interestingly, the PROMIS Fatigue 7-item instrument and the one-item SCL-Fatigue question had similar magnitudes of association with the other outcome scores. We observed a direct, linear relationship between worsening of PROMIS Fatigue T-scores and SCLFatigue levels from 'None' through 'Extremely' bothersome (Figure S2).

\section{5 | Bivariate associations between patient-reported outcomes and HBV laboratory and clinical markers}

Bivariate correlations between the four outcome scores and all HBV covariates were almost nonexistent to small (range: $r=0.0$ to 0.20 ) 
FIGURE 3 Composition of symptoms ranging from 'None' to 'Extremely'. Subcategories with less than $1 \%$ are not labelled in the figure

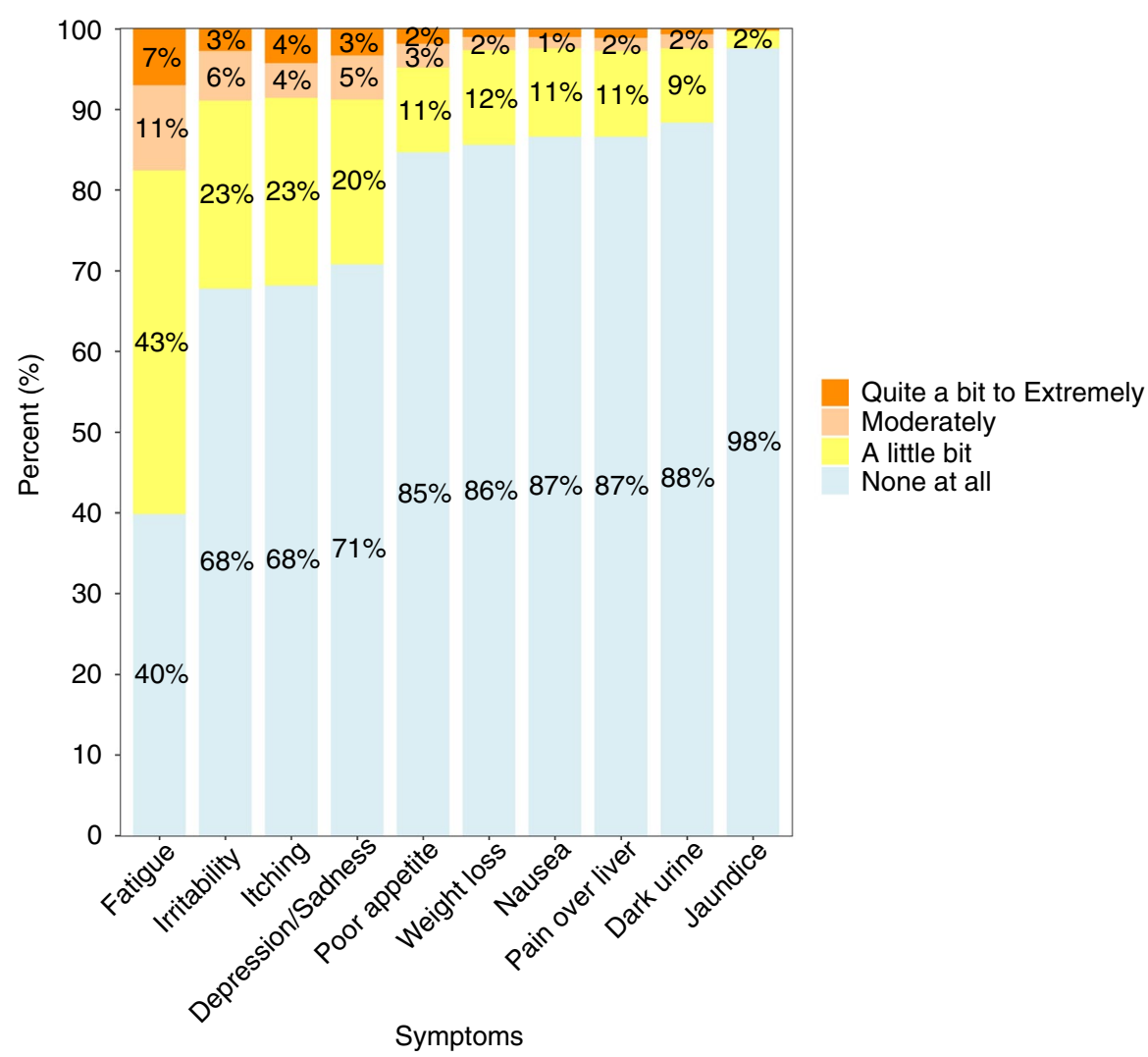

(Figure S3). HBV phenotype, $\mathrm{HBeAg}$ and HBV DNA did not correlate significantly with any of the outcome measures. Apart from the small correlations between PCS and a higher number of comorbidities and medications ( $r=0.20$ ), all other correlations with HBV laboratory tests or clinical markers were negligible.

\subsection{Unadjusted and adjusted associations between patient-reported outcome measures with HBV disease markers and demographic characteristics}

Unadjusted associations between each outcome score and demographics, clinical and laboratory covariates are provided in Table S1. Female sex, lower education, abnormal AST, APRI >1.5 and a higher number of comorbidities were most frequently associated with worse outcome scores.

For the multivariable analyses, we first accounted for sociodemographics (sex, age, race, education), then used stepwise regression models for variable selection from the following covariates: HBV phenotype, ALT, AST, HBeAg, HBV DNA, albumin, APRI, cirrhosis, comorbidities and concomitant medications. APRI and a higher number of comorbidities independently predicted PROMIS Fatigue and the PCS score (See Table 2). APRI was selected for T-SCL. None of the clinical variables were selected for MCS. ${ }^{1}$

${ }^{1}$ Since APRI scores are calculated using AST and platelet count, we investigated removal of APRI from the models and adding platelet count. Abnormal AST was selected for three of four PROs. However, the original multivariable model including APRI was still a better fit for the data, therefore, the remaining results are based on the original modelling plan.
Participants who had APRI $>1.5$ reported 7.73 points worse Fatigue (95\% Cl: 3.89, 11.56), 9.31 points worse PCS (95\%Cl: $-12.56,-6.06)$ and $165 \%$ worse total symptom burden (mean ratio: 2.65 ; $95 \% \mathrm{Cl}: 1.54$, 4.56) compared to those with APRI $\leq 0.5$. Participants who had two or more comorbidities reported 3.02 points worse Fatigue $(95 \% \mathrm{Cl}$ : 0.99 , 5.06) and 3.97 points worse PCS scores ( $95 \% \mathrm{Cl}$ : $-5.70,-2.25)$.

A few sociodemographic variables were also associated with worse outcome scores (Table 2). Females had 3.16 point worse Fatigue (95\% Cl: 2.02, 4.30), 1.93 points worse $\mathrm{MCS}(95 \% \mathrm{Cl}:-3.23$, $-0.64)$, 0.98 points worse PCS (95\% Cl: $-1.94,-0.02)$ and $30 \%$ worse total symptom burden (mean ratio: $1.30 ; 95 \% \mathrm{Cl}: 1.09,1.55)$ compared to males. Compared to Asians, Black and White patients reported $37 \%$ (mean ratio: $1.37 ; 95 \% \mathrm{Cl}: 1.05,1.78$ ) and $38 \%$ (mean ratio: 1.38 ; $95 \% \mathrm{Cl}: 1.06,1.82)$ worse total symptom burden. Participants with lower education had 1.61 points worse Fatigue $(95 \% \mathrm{Cl}: 0.43,2.79)$ and 1.23 points worse PCS scores $(95 \% \mathrm{Cl}$ : $-2.23,-0.23)$. Collectively, the covariates selected in each regression model only explained $4 \%$ of the variation in mental health, $17 \%$ of the variation in physical health, $10 \%$ of the variation in Fatigue, and $7 \%$ of the variation in total symptom burden.

\section{7 | Exploratory analyses of symptoms predicting physical and mental health}

Given the low explanatory power of the original regression models to predict PCS and MCS, yet the moderate correlations observed between symptom scores and PCS and MCS, we speculated that adding 
TAB LE 2 Multivariable models for fatigue, mental component summary score, physical component summary score, and total symptom score $(n=707)$

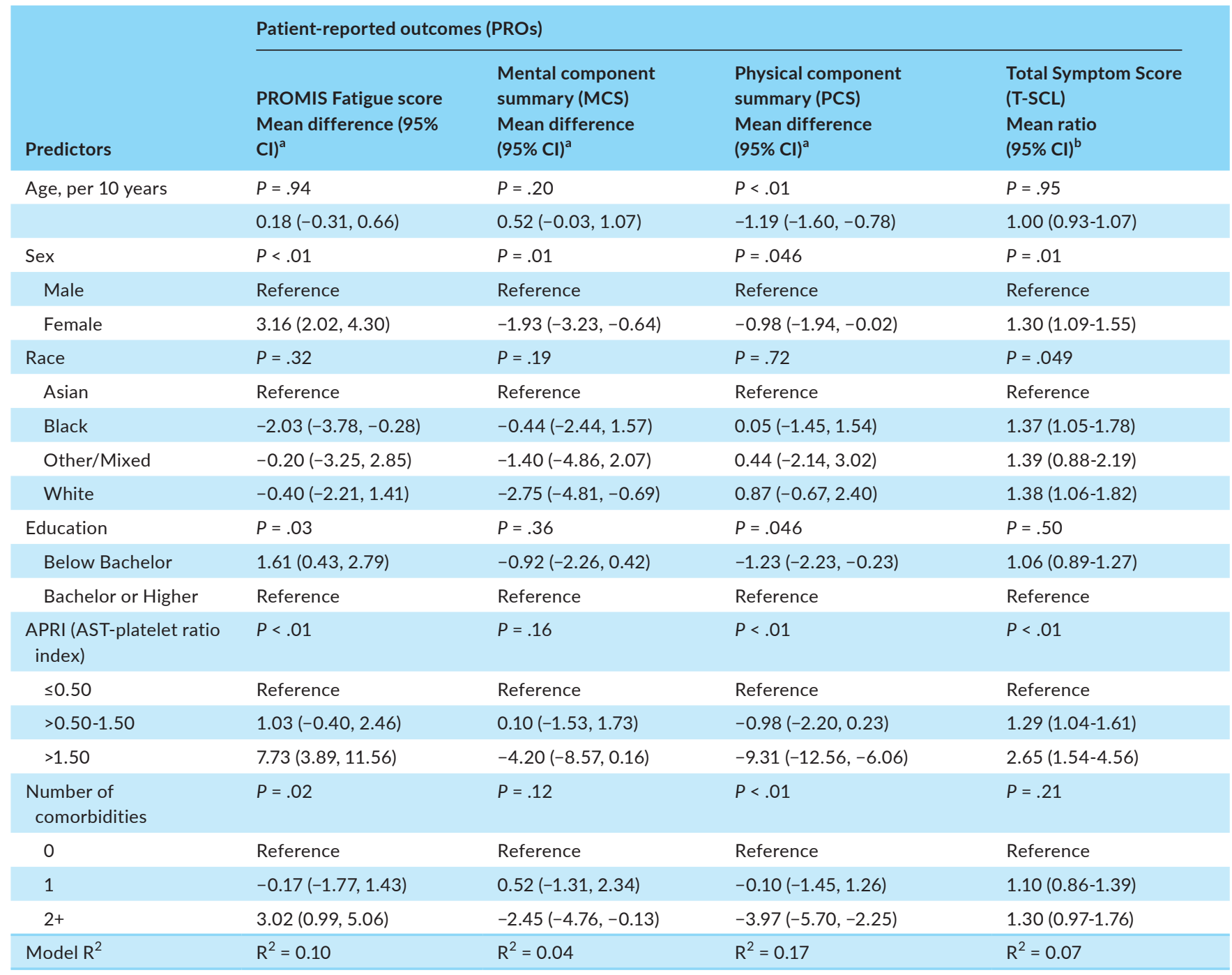

Note: $P$ values were adjusted for multiple comparisons across four patient-reported outcomes using the Bonferroni-Holm method.

${ }^{a}$ Multiple linear regression was used to estimate the adjusted association between each predictor and each patient-reported outcome respectively. If a 95\% confidence interval for a mean difference does not include 0, the mean of Fatigue, Mental Component Summary score, Physical Component Summary score is significantly different between groups or per unit change in a continuous predictor. $\mathrm{R}^{2}$ is the proportion of the response patient reported outcome variable variation that is explained by a linear model.

${ }^{\mathrm{b}}$ Negative binomial regression was used to estimate the adjusted association between each predictor and total symptom score. If a $95 \%$ confidence interval ( $\mathrm{Cl}$ ) for a mean ratio does not include 1, the mean of T-SCL is significantly different between groups or per unit change in a continuous predictor.

T-SCL to the regression models might help explain additional variability in MCS and PCS scores, over and above the initial set of covariates. Table 3 shows that T-SCL independently explained $41 \%$ of the variation in MCS, totalling 44\% explanatory power when added to the other covariates in the model. Likewise, T-SCL independently explained $22 \%$ of the variation in PCS scores, totalling $34 \%$ of the variation in PCS when added to the original covariates in the model. Thus, T-SCL improved the prediction of MCS from $4 \%$ to $44 \%$ and PCS from $17 \%$ to $34 \%$ when added to the original models. We also examined the unique contribution of each symptom to the original prediction models (Table 3). Most notably, the Depression/Sadness item from the SCL predicted additional $50 \%$ of the variation in MCS scores. For every categorical increase in depression/sadness (eg from 'A little bit' to 'Moderately'), a patient's MCS score decreases by 8.10 points. For PCS, the SCL items for Fatigue, Nausea and Pain over the Liver each contributed 10\%-14\% of additional variation to the orginal models. For example, for every categorical increase in Fatigue (eg from 'Moderately' to 'Quite a Bit'), a patient's PCS score decreased by 2.83 points.

\subsection{Assessment of HBV disease activity and stage}

Assessment of disease severity focuses upon two separate features: disease activity (grade) and disease stage (fibrosis). ${ }^{20}$ Disease 
activity is the degree of inflammation, and can worsen, improve or resolve. Disease stage is reflected in degree of fibrosis and cirrhosis, factors that usually progress and do not resolve. We aimed to understand how much symptoms or health-related quality of life may be associated with disease activity and disease stage. Using ALT and AST to reflect disease grade and APRI to reflect disease stage, we generally found that outcomes were worse with more advanced stages of disease (APRI >1.50) and affected by more severe disease activity, and generally only at the extremes of both. The different ranges of the 10 symptoms at different levels of APRI are shown in Figure S4 and at different levels of ALT in Figure S5. Thus, the 10 symptoms correlated with APRI, but the association was largely with APRI >1.50 and with symptoms that were 'Moderate' or worse, rather than 'A little bit'. Similarly, ALT elevations correlated with symptoms, but only at the extremes of ALT ( $>5$ upper limit of normal) and with 'Moderate' or worse symptoms, as opposed to 'A little bit'. Thus, symptoms described as 'A little bit' were generally nonspecific and were not more frequent with more severe disease activity or advanced stage. In contrast, describing symptoms as 'Moderately', 'Quite a Bit' or 'Extremely' bothersome was associated with more severe disease activity or advanced stage of disease.

\section{4 | DISCUSSION}

Findings from previous studies of quality of life in patients with HBV have been difficult to extrapolate to patients living in the United States and Canada due to underlying sociodemographic, regional and cultural influences that may exert strong effects on HRQoL scores. Additionally, symptom burden associated with HBV has been understudied, but may be important to understanding patients' experiences with living with $\mathrm{HBV}$, a highly infectious and frequently stigmatising liver disease. In this cross-sectional analysis, we included a large multiethnic North American cohort from 21 adult liver centres across the United States and Toronto. This cohort was predominantly of Asian ethnicity, college educated, asymptomatic and healthy; a very low proportion had cirrhosis $(<1 \%)$, multiple comorbidities or were taking any medications (<25\%). Nevertheless, interesting findings were observed. On average, this relatively healthy cohort without severe liver disease experienced very few HBV-related symptoms and had uncompromised quality of life. Secondly, HBV virologic markers such as HBV DNA, quantitative HBsAg and HBV phenotypes were not independently associated with symptoms or quality of life. High APRI score, reflecting more advanced liver disease stage was the only important clinical variable significantly associated with worse symptoms and quality of life. Finally, we discovered a robust relationship between symptoms and physical and mental quality of life, highlighting the importance of measuring HBV-related symptoms in future research and in clinical practice, as some symptoms may play a crucial role in the mental and physical well-being of patients living with HBV.

This North American sample had, on average, equal or better HRQoL and fatigue scores compared to US general population norms. This finding may be due to characteristics of the cohort such as absence of cirrhosis, fewer comorbidities or concomitant medications, and higher levels of education and employment compared to population norms. ${ }^{21}$ However, our findings are consistent with one Canadian study ${ }^{10}$ and one recent international Phase II clinical trial ${ }^{22}$ which found no differences in HRQoL between noncirrhotic HBV patients and population norms. Although few studies have investigated symptoms as a primary endpoint, our findings are consistent with a Persian study which also found a low prevalence of symptoms and mostly reported as mild and nondistressing. ${ }^{3}$

High APRI score was associated with worse PROs scores. This finding is consistent with several other studies documenting worse PROs in patients with more severe liver disease. ${ }^{2,3,5,23}$ However, it should be noted that APRI is most sensitive for cirrhosis detection in patients with chronic hepatitis $\mathrm{C}$ or non-alcoholic fatty liver disease, compared to HBV patients due to a greater degree of variability in untreated HBV patients. ${ }^{24}$ While the definitions of advanced liver disease (eg compensated, decompensated, hepatocellular carcinoma, Child-Pugh scores) have varied among studies, most have shown worse HRQoL and symptoms among patients with decompensated cirrhosis or liver cancer. In the current study, less than $1 \%$ of patients were diagnosed with cirrhosis by expert adjudication and only $2 \%$ had APRI scores $>1.50$. These patients experienced five symptoms (on average) and many experienced moderate to severe fatigue, irritability, pruritus and depression (Figure S4). Additionally, participants with elevated ALT (>5 upper limit of normal) also experienced more symptoms (Figure S5). Therefore, clinicians should suspect advanced liver disease or high disease activity in patients complaining of moderate to extremely bothersome symptoms.

While none of the other HBV laboratory or clinical markers were associated with PROs, we identified other factors that identify patient subgroups at risk for worse PROs. Patients with more comorbidities may be at risk for worse fatigue and physical health, a finding consistent with other studies. ${ }^{3,10,25}$ Female patients are also at risk for worse fatigue, mental health, physical health and report $30 \%$ more symptoms, compared to men; also consistent with prior studies. ${ }^{9,22}$ We also discovered that Black and White patients report approximately $40 \%$ more symptoms than Asians and that lower education is associated with more fatigue and worse physical health. Taken together, these data suggest that future research endeavours which seek to examine factors associated with HRQOL and symptoms in HBV populations should include advanced liver disease, number of comorbidities and certain sociodemographics in statistical modelling. ${ }^{26}$ Equally important to recognise, however, is that collectively these factors explained very little variation in self-reported physical (17\%) and mental health (4\%), making it incumbent upon future investigations to evaluate additional factors that might help explain physical and mental health in HBV populations; for instance, other HBV studies have found age, sex, income level, body mass index and psychiatric comorbidity predictive of HRQoL scores. ${ }^{2,3,10,22,25}$

Symptom burden has rarely been explored in HBV patients and none in North American cohorts. ${ }^{3,14,27}$ Given this shortcoming, we explored the utility of adding symptom burden (T-SCL score) and individual symptom scores to predict mental and physical health. 


\begin{tabular}{|c|c|c|c|c|}
\hline \multirow[b]{2}{*}{ Models } & \multicolumn{2}{|l|}{$\begin{array}{l}\text { Mental component } \\
\text { summary (MCS) }\end{array}$} & \multicolumn{2}{|l|}{$\begin{array}{l}\text { Physical component } \\
\text { summary (PCS) }\end{array}$} \\
\hline & $\begin{array}{l}\text { Mean difference } \\
(95 \% \mathrm{Cl})^{\mathrm{a}}\end{array}$ & $\mathrm{R}^{2}$ & $\begin{array}{l}\text { Mean difference } \\
(95 \% \mathrm{Cl})^{\mathrm{a}}\end{array}$ & $\mathrm{R}^{2}$ \\
\hline Multivariable model $(\mathrm{MV})^{\mathrm{b}}$ & - & 0.04 & - & 0.17 \\
\hline $\mathrm{MV}+\mathrm{T}-\mathrm{SCL}$ & $-1.46(-1.59,-1.33)$ & 0.44 & $-0.77(-0.88,-0.65)$ & 0.34 \\
\hline MV + SCL-Fatigue & $-4.91(-5.50,-4.33)$ & 0.31 & $-2.83(-3.30,-2.36)$ & 0.31 \\
\hline MV + SCL-Irritability & $-6.05(-6.74,-5.35)$ & 0.33 & $-2.30(-2.90,-1.71)$ & 0.23 \\
\hline MV + SCL-Itching & $-2.99(-3.75,-2.23)$ & 0.12 & $-1.97(-2.54,-1.40)$ & 0.22 \\
\hline $\begin{array}{l}\text { MV + SCL-Depression/ } \\
\text { Sadness }\end{array}$ & $-8.10(-8.68,-7.52)$ & 0.54 & $-2.10(-2.71,-1.50)$ & 0.22 \\
\hline MV + SCL-Poor appetite & $-5.36(-6.35,-4.37)$ & 0.18 & $-2.79(-3.55,-2.02)$ & 0.23 \\
\hline MV + SCL-Weight loss & $-2.67(-3.90,-1.44)$ & 0.07 & $-1.58(-2.50,-0.66)$ & 0.18 \\
\hline MV + SCL-Nausea & $-4.92(-6.13,-3.71)$ & 0.12 & $-4.65(-5.53,-3.77)$ & 0.28 \\
\hline $\mathrm{MV}+\mathrm{SCL}$-Pain over liver & $-4.80(-5.98,-3.62)$ & 0.12 & $-4.36(-5.22,-3.50)$ & 0.27 \\
\hline $\mathrm{MV}+\mathrm{SCL}-$ Dark urine & $-5.40(-6.82,-3.97)$ & 0.11 & $-4.24(-5.29,-3.18)$ & 0.24 \\
\hline MV + SCL-Jaundice & $-6.19(-8.80,-3.58)$ & 0.07 & $-2.90(-4.86,-0.94)$ & 0.18 \\
\hline
\end{tabular}

TABLE 3 Associations between symptoms and Mental Component Summary score (MCS) and Physical Component Summary score (PCS) $(\mathrm{n}=707)$

${ }^{a}$ Generalized linear regression was used to estimate the association between predictors and each patient-reported outcome. If a $95 \%$ confidence interval does not include 0 , there is a significantly difference in the mean of the mental or physical component summary score, per one-unit increase in total symptom burden or per one-level increase in each symptom checklist symptom. $R^{2}$ is the proportion of the response in mental or physical component summary score variation that is explained by a linear model.

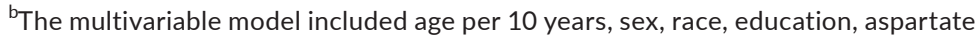
aminotransferase-platelet-ratio index (APRI), and comorbidities.

A well-known conceptual model by Wilson and Cleary describes a causal model whereby biological causes (eg virus and cirrhosis) may lead to physical or mental symptoms (defined as a 'patient's perception of an abnormal physical, emotional or cognitive state'), which in turn, affects functioning (physical, social, role,and psychological), health perceptions and overall quality of life ${ }^{28}$ Consistent with this model, we discovered that when the T-SCL score was added to the initial regression models, we were able to substantially increase prediction of mental health from $4 \%$ to $44 \%$. Moreover, one item from the SCL instrument ('How bothered are you by depression or sadness') predicted mental health functioning better $\left(R^{2}=0.54\right)$ than the complete 10-item survey. Future studies seeking to understand mental health status should include depression/sadness symptom measurement which will have clinical implications for patient care. Likewise, adding T-SCL to the models to predict self-reported physical health QOL improved predictability from $17 \%$ to $34 \%$.

Interestingly, both the 7-item PROMIS Fatigue and the oneitem SCL-Fatigue were equally correlated with mental health status and with most individual symptoms, suggesting that they might perform equally well in future studies. The advantage of the PROMIS ${ }^{\circledR}$ Fatigue instrument is that these measures were rigorously developed and validated for use across general and medical populations. ${ }^{16,17}$ However, a one-item fatigue question in a 10 -item multi-symptom instrument might also be attractive when constrained by time or resources. Subsequent studies might explore the psychometric properties of the National Institute of Diabetes and Digestive and Kidney Diseases Symptom Checklist for use in the HBV population.

This is the largest, most comprehensive assessment of patient-reported outcomes in a population of patients with HBV; nonetheless the study has limitations. Considering the low proportion of cirrhotic patients, our findings cannot be generalised to patients with more severe liver disease. The low rate of cirrhosis in this cohort may in part reflect the requirement of enrolling only untreated patients into the HBRN cohort study. ${ }^{15}$ As HBV therapy is recommended only in patients who have suspected moderate inflammation or fibrosis, it is not surprising that this untreated cohort had milder liver disease. In addition, the sensitivity and specificity of the APRI score for detecting advanced fibrosis in HBV patients are limited. ${ }^{24}$ Furthermore, a more accurate assessment of disease staging such as liver elastography or liver biopsy was not undertaken prior to or at the same visit that these four PROs were collected in the majority of patients (only $4 \%$ and $11 \%$ had undergone these procedures respectively). The PROMIS Fatigue survey was not collected in over 350 non-English/ non-Spanish speaking patients because validated translations of this instrument were not available at study initiation. Finally, North American population controls of Asian ethnicity, the main ethnic group in our HBV cohort, were not available for the PROMIS ${ }^{\circledR}$ or SF-36 instruments, but it is possible that these patients have better PRO scores compared to population controls. Future studies should be conducted to evaluate the relevance and understandability of these patient-reported outcomes measures in order to adapt them 
for use in different ethnic populations such as this predominantly Asian population living the United States. Finally, other factors that might explain decrements in HRQoL were not collected (eg feeling of social stigma, sleep disturbance and health-related anxiety).

To conclude, untreated North American patients with HBV and generally mild liver disease report favourable HRQoL scores and minimal HBV-associated symptoms. Except for the presence of advanced liver disease, HBV disease or laboratory markers did not affect patients' well-being, functioning, fatigue or other symptoms. The presence of moderate to extreme symptoms, especially fatigue, irritability, itch and depression/sadness may also signal the presence of advanced liver disease. As HBV is a dynamic disease, longitudinal studies are needed to better understand the evolution of symptoms and HRQoL with the natural history of HBV and also in the setting of HBV therapy.

\section{ACKNOWLEDGEMENTS}

In addition to the authors, the HBRN acknowledge the contributions of the following: Harvard Consortium: Jianghe Niu, PhD, Asad Javaid, MBBS, Bilal Nasir, MBBS, Ammu Susheela, MBBS, Imad Nasser, MD (Beth Israel Deaconess Medical Center, Boston, MA), Arley Donovan, Nifasha Rusibamayila, Cara Foley (Massachusetts General Hospital, Boston, MA). Minnesota Alliance for Research in Chronic Hepatitis B: Alisha C. Stahler, Linda Stadheim, RN (Mayo Clinic Rochester, Rochester, MN), John Lake, MD, Philip Lacher (University of Minnesota, Minneapolis, MN). Midwest Hepatitis B Consortium: Kathryn Rushing, RN (Saint Louis University School of Medicine, St Louis, MO), Debra DeMarco Shaw, RN, BSN, Lisa Kessels, RN, Michael K. Klebert, PhD, RN, ANP-BC (Washington University School of Medicine, St. Louis, MO). University of Toronto Consortium: Seham Noureldin, PhD, Danie La, RN, Lucie Liu, MSc, CCRP, Diana Kaznowski, RN, Jiayun Chen, Fengfei Huang, Doinita Vladutu, Orlando Cerocchi (Toronto General Hospital, Toronto, Ontario). HBV CRN North Texas Consortium: Debra Rowan, LVN (Division of Digestive and Liver Diseases, University of Texas Southwestern Medical Center at Dallas, Dallas, TX), Sheila Bass (University of Texas Southwestern, Dallas, TX), Barbara Lilly, BS (Baylor University Medical Center, Dallas, TX). Los Angeles Hepatitis B Consortium: Samuel French, MD, Velma Peacock, RN (David Geffen School of Med, UCLA, Los Angeles, CA). San Francisco Hepatitis B Research Group Consortium: Marion Peters, MD, Ashley Shobe, MS, Rayshawnda Davis, Romuald Kuras, Claudia Ayala, MS, Ivy Lau, BS (University of California-San Francisco, San Francisco, CA), Veronika Podolskaya, BS, NCPT, Anna von Bakonyi, LVN, CCRC, Nata DeVole, RN (California Pacific Medical Center Research Institute, San Francisco, CA). Michigan Hawaii Consortium: Barbara McKenna, MD, Karen Choi, MD, Kelly Oberhelman, PAC, Sravanthi Kaza, Bpharm, Isabel Moran (University of Michigan, Ann Arbor, MI), Leslie Huddleston, NP, Richmond Wong (The Queen's Medical Center, University of Hawaii, Honolulu, HI). Chapel Hill, NC Consortium: A. Sidney Barritt, M.D. , Tiffany Marsh, BA, Vikki Metheny, ANP, Danielle Cardona, PA-C (University of North Carolina at Chapel Hill, Chapel Hill, NC). Virginia Commonwealth University Medical Center: Paula G. Smith, RN, BSN, Charlotte Hofmann, RN (Virginia Commonwealth University Health
System, Richmond, VA). PNW/Alaska Clinical Center Consortium: Alycia Wolfstone, RN, MN (University of Washington Medical Center, Seattle, WA) Jody Mooney, Lupita Cardona-Gonzalez (Virginia Mason Medical Center, Seattle, WA). Liver Diseases Branch, NIDDK, NIH: Nancy Fryzek, RN, BSN, Elenita Rivera, BSN, Nevitt Morris, Vanessa HaynesWilliams, Amy Huang, RN, Catherine Nadal, RN, MS, Jaha NormanWheeler, RN, BA (National Institutes of Health, Bethesda, MD). Liver Disease Research Branch, NIDDK, NIH: Averell H. Sherker, MD, Rebecca J. Torrance, RN, MS, Sherry R. Hall, MS (National Institutes of Health, Bethesda, MD). Immunology Center: Mary E. Valiga, RN, Keith Torrey, BS, Danielle Levine, BS, James Keith, BS, Michael Betts, PhD (University of Pennsylvania, Philadelphia, PA), Luis J. Montaner, DVM, DPhil (Wistar Institute, Philadelphia, PA). Data Coordinating Center: Frani Averbach, MPH, Tamara Haller, Regina Hardison, MS, Stephanie Kelley, MS, Christina Lalama, MS, Sharon Lawlor, MBA, Manuel Lombardero, MS, Andrew Pelesko, BS, Donna Stoliker, Melissa Weiner, MPH, Ella Zadorozny, MS, Qian Zhao, PhD (Graduate School of Public Health, University of Pittsburgh, Pittsburgh, PA).

Declaration of personal Interests: Donna M. Evon receives research grant funding (to UNC) from Gilead Sciences Inc and Merck Sharp and Dohme. Michael Fried has received research funding from and served as a consultant for AbbVie, BMS, Gilead and Merck, and TARGET PharmaSolutions. Stock in TARGET PharmaSolutions is held in an independently managed trust. Mandana Khalili received research grant funding (to her institution) from Gilead Sciences Inc and Intercept Pharmaceutical and has served as a scientific consultant for Gilead Science. Robert Fontana has received research support from Gilead, BMS and Abbvie and provides consulting to Alynam. Colina Yim has received speakers honorarium from Abbvie Canada, and consulting fees from Abbvie Canada and Lupin Pharma Canada. Hsing-Hua S. Lin, Abdus S. Wahed and Jay H. Hoofnagle have no conflict of interests to disclose.

\section{AUTHORSHIP}

Guarantor of the article: Dr. Donna M. Evon.

Author contributions: Donna M. Evon, Hsing-Hua S. Lin, Mandana Khalili, Robert J. Fontana, Colina Yim, Abdus S. Wahed, Michael W. Fried, and Jay $\mathrm{H}$. Hoofnagle performed the research, analysed the data, and contributed to writing the manuscript. Mandana Khalili, Robert J. Fontana, Colina Yim, and Michael W. Fried assisted with data collection. Donna M. Evon, Hsing-Hua S. Lin, Abdus S. Wahed, and Jay $\mathrm{H}$. Hoofnagle designed the research study, and contributed to the design of the current study.

All authors have approved the final version of this manuscript.

\section{ORCID}

Donna M. Evon (iD https://orcid.org/0000-0002-1414-1846

Mandana Khalili iD https://orcid.org/0000-0001-9178-9139

\section{REFERENCES}

1. Terrault NA, Lok ASF, McMahon BJ, et al. Update On Prevention, Diagnosis, And Treatment Of Chronic Hepatitis B: AASLD 2018 Hepatitis B Guidance. Hepatology. 2018;67:1560-1599. 
2. Modabbernia A, Ashrafi M, Malekzadeh R, Poustchi H. A review of psychosocial issues in patients with chronic hepatitis B. Arch Iran Med. 2013;16:114-122.

3. Saffari M, Alavian SM, Naderi MK, Pakpour AH, Al Zaben F, Koenig HG. Cross-cultural adaptation and psychometric assessment of the liver disease symptom index 2.0 to measure health-related quality of life among iranian patients with chronic hepatitis B. J Transcult Nurs. 2016;27:496-508.

4. Dan AA, Kallman JB, Wheeler A, et al. Health-related quality of life in patients with non-alcoholic fatty liver disease. Aliment Pharmacol Ther. 2007;26:815-820.

5. Ong SC, Mak B, Aung MO, Li SC, Lim SG. Health-related quality of life in chronic hepatitis B patients. Hepatology. 2008;47:1108-1117.

6. Lam ET, Lam CL, Lai CL, Yuen MF, Fong DY. Psychometrics of the chronic liver disease questionnaire for southern chinese patients with chronic hepatitis B virus infection. World J Gastroenterol. 2009;15:3288-3297.

7. Altindag A, Cadirci D, Sirmatel F. Depression and health related quality of life in non-cirrhotic chronic hepatitis B patients and hepatitis B carriers. Neurosciences. 2009;14:56-59.

8. Tan NC, Cheah SL, Teo EK, Yang LH. Patients with chronic hepatitis B infection: what is their quality of life? Singapore Med J. 2008;49:682-687.

9. Lam ET, Lam CL, Lai CL, Yuen MF, Fong DY, So TM. Health-related quality of life of Southern Chinese with chronic hepatitis B infection. Health Qual Life Outcomes. 2009;7:52.

10. Woo G, Tomlinson G, Yim C, et al. Health state utilities and quality of life in patients with hepatitis B. Can J Gastroenterol. 2012;26:445-451.

11. Kim SJ, Han KT, Lee SY, Park EC. Quality of life correlation with socioeconomic status in Korean hepatitis-B patients: a cross sectional study. Health Qual Life Outcomes. 2015;13:55.

12. Di Bisceglie AM, Lombardero $M$, Teckman J, et al. Determination of hepatitis $B$ phenotype using biochemical and serological markers. J Viral Hepat. 2017;24:320-329.

13. Evon DM, Wahed AS, Johnson G, et al. Fatigue in patients with chronic hepatitis B living In North America: results from the hepatitis B research network (HBRN). Dig Dis Sci. 2016;61:1186-1196.

14. Jang $\mathrm{Y}$, Boo S, Yoo $\mathrm{H}$. hepatitis B virus infection: fatigue-associated illness experiences among Koreans. Gastroenterol Nurs. 2018;41:388-395.

15. Ghany MG, Perrillo R, Li R, et al. Characteristics of adults in the hepatitis $B$ research network in North America reflect their country of origin and hepatitis B virus genotype. Clin Gastroenterol Hepatol. 2015;13:183-192.

16. Cella D, Riley W, Stone A, et al. The patient-reported outcomes measurement information system (promis) developed and tested its first wave of adult self-reported health outcome item banks: 2005-2008. J Clin Epidemiol. 2010;63:1179-1194.

17. Reeve BB, Hays RD, Bjorner JB, et al. Psychometric evaluation and calibration of health-related quality of life item banks: plans for the patient-reported outcomes measurement information system (PROMIS). Med. Care. 2007;45:S22-S31.
18. Ware JE Jr. SF-36 health survey update. Spine. 2000;25:3130-3139.

19. Ware JE Jr, Snow KK, Kosinski M, Gandek B. SF-36 Health Survey Manual And Interpretation Guide. Boston, MA: New England Medical Center, The Health Institute; 1993.

20. Desmet VJ, Gerber M, Hoofnagle JH, Manns M, Scheuer PJ. Classification of chronic hepatitis: diagnosis grading and staging. Hepatology. 1994;19:1513-1520.

21. Schiller JS, Lucas JW, Ward BW, Peregoy JA. Summary health statistics for U.S. adults: national health interview survey, 2010. Vital Health Stat 10. 2012;252:1-207.

22. Younossi ZM, Stepanova M, Janssen HLA, et al. Effects of treatment of chronic hepatitis $B$ virus infection on patient-reported outcomes. Clin Gastroenterol Hepatol. 2018;16 (1641-1649):E1646.

23. Bondini S, Kallman J, Dan A, et al. Health-related quality of life in patients with chronic hepatitis B. Liver Int. 2007;27:1119-1125.

24. Sebastiani G, Castera L, Halfon P, et al. The impact of liver disease aetiology and the stages of hepatic fibrosis on the performance of non-invasive fibrosis biomarkers: an international study of 2411 cases. Aliment Pharmacol Ther. 2011;34:1202-1216.

25. Karacaer Z, Cakir B, Erdem H, et al. Quality of life and related factors among chronic hepatitis B-infected patients: a multi-center study. Health Qual Life Outcomes. 2016;14:153.

26. Teuber G, Schäfer A, Rimpel J, et al. Deterioration of health-related quality of life and fatigue in patients with chronic hepatitis C: association with demographic factors, inflammatory activity and degree of fibrosis. J Hepatol. 2008;49:923-929.

27. Simonetti G, Gitto S, Golfieri L, et al. Quality of life of hepatitis $B$ virus surface antigen-positive patients with suppressed viral replication: comparison between inactive carriers and nucleot(s)ide analog-treated patients. Eur J Gastroenterol Hepatol. 2018;30:14-20.

28. Wilson IB, Cleary PD. Linking clinical variables with health-related quality of life. a conceptual model of patient outcomes. JAMA. 1995;273:59-65.

\section{SUPPORTING INFORMATION}

Additional supporting information will be found online in the Supporting Information section.

How to cite this article: Evon DM, Lin H-HS, Khalili M, et al; for the Hepatitis B Research Network (HBRN). Patient-reported outcomes in a large North American cohort living with chronic hepatitis B virus: a cross-sectional analysis. Aliment Pharmacol Ther. 2020;51:457-468. https://doi.org/10.1111/apt.15618 